\title{
Computer-assisted tkr vs conventional tkr: post- operative ankle radiographic finding and ankle clinical assessment
}

\begin{abstract}
Background: Few previous studies showed that the conventional total knee replacement (TKR) has affection to the same side of talar tilt (TT). We expected to prevent this problem by the computer-assisted (CAS) TKR.

Objective: The purpose of this study was to compare between pre and post-operative talar tilt and ankle clinical assessment on the CAS TKR and the Conventional TKR in 28 patients (56 knees) whom underwent bilateral TKR.

Material and Method: 28 patients, 56 knees, whom underwent both CAS total knee replacement (TKR) and conventional total knee replacement (TKR), in both knees, with the combination of Gap Balance and Measurement Resection techniques performed by one surgeon (P. Sriphirom) at Rajavithi Hospital, Bangkok. The post-operative has a 12 months follow-up for ankle radiographic finding by tibiotalar angle (TTA), tibial articular surface angle (TAS), and talar tilt (TT) $=($ TAS-TTA) and for ankle clinical assessment by foot functional index (FFI) from pre-operation and post-operation from both groups. The study also compares the CAS TKR with the Conventional TKR for pre-operation and postoperation.
\end{abstract}

Results: 56 knees, 28 patients, mean age $=67.79$ years whom underwent bilateral TKR by the Conventional group and the CAS group had pre-operative TT (TT = TAS - TTA). The Conventional group $=1.5(-5,8)$, the CAS group $=.5(-5,8), \mathrm{P}$ value $=.65$. On post-operative TT the Conventional group $=.0(-5,3)$, the CAS group $=1.0(-3,8)$, the $\mathrm{P}$ value $=.4$. The comparison of pre-operative TT and post-operative TT in the Conventional group, the P value $=.01$. On pre-operative TT and post-operative TT in the CAS group, the P value $=.65$. TT was significantly different in the Conventional group but was not significantly different in the CAS group. The ankle clinical assessment by foot functional index (FFI), which is (1) Pain, (2) Difficulty living, and (3) Daily life activity limitation. The pre-operative FFI in the Conventional group $=1.85(.81,6.88)$ and pre-operative FFI in the CAS group $=$ $1.91(.24,66.5)$, the $\mathrm{P}$ value $=.57$. The post-operative FFI in the Conventional group $=$ $1.68(0.24,7.0)$ and post-operative FFI in the CAS group $=1.65(.24,6.76)$, the P value $=.04$, which showed a significantly different between the post-operative FFI from both groups. In the Conventional group the post-operative FFI was not significantly different from pre-operative FFI, the $\mathrm{P}$ value $=.2$ but for the CAS group the post-operative FFI was not significantly different from pre-operative FFI, the $\mathrm{P}$ value $=.04$.

Conclusion: This study has shown that the conventional TKR effected to post-operative talar tilt but the CAS TKR has less effect and was not significantly different to ankle joint. Finally, the study needs to be conducted on more patients and to be observed on a longer term follow-up.

Keywords: Total knee arthroplasty, TKR, CAS TKR, Ankle score
Volume 6 Issue 7 - 2016

\author{
Methee Khongphaophong,' Thakrit \\ Chompoosang, ${ }^{2}$ Pornpavit Sripirom, ${ }^{2}$ \\ Phattara Chunsiri \\ 'Department of Orthopaedic, Samut Sakhon Hospital, Thailand \\ ${ }^{2}$ Department of Orthopaedic, Rajavithi Hospital, College of \\ Medicine, Rangsit University, Thailand
}

Correspondence: Methee Khongphaophong, Department of Orthopaedic, Samut Sakhon Hospital, Samut Sakhon, Thailand 7400,Tel 6680323660 Email metheek@gmail.com

Received: April 19, 2016 | Published: December 30, 2016

\section{Introduction}

When osteoarthritis causes severe varus and valgus knee deformities, it is often also present in the ankle joint. ${ }^{1}$ and it is not rare to find those who have undergone total knee arthroplasty presenting with ankle osteoarthritis. Many of them complain of pain in the ankle joint following total knee arthroplasty, and in some cases radiographs show progressive degenerative changes in the ankle.

Deformity of OA knee changes normal mechanical axis and may also change the axis of rotation of the ankle joint. ${ }^{2}$ Recently, Jung Hee Lee at al. found that after total knee arthroplasty, arthritis developed or progressed radiographically in many patients' ankles. ${ }^{3}$

As yet, there is no single recommended technique for minimizing postoperative ankle arthritis change in total knee arthroplasty (TKA).
This study compared and evaluated computer-assisted total knee arthroplasty (CAS-TKA) and conventional total knee arthroplasty (TKA), and we anticipated that computer- assisted total knee arthroplasty might minimize the postoperative ankle effect both clinically and radiographically.

\section{Materials and methods}

We retrospectively enrolled 28 patients who underwent traditional total knee arthroplasty on one knee and computer-assisted total knee arthroplasty on the other with a 6-month interval between operations. All patients attended for follow-up for at least 12 months between February 2010 and November 2012 in our institute. There were 26 females and 2 males, their mean age was 67.9, and all cases were 1o OA knee. We excluded post-traumatic knee or ankle joint, $20 \mathrm{OA}$ 
(inflammatory, infection, congenital), and valgus knee. We performed pre-operative and postoperative scannograms in all cases for tibial articular surface angle (TAS), tibio-talar angle (TTA) and talar tilt (TT). ${ }^{1}$ (Figure 1) after which we calculated Talar tilt (TT), for which the normal range is $(-4,+4)$, as the difference between TAS and TTA for radiologic ankle assessment. We compared the results of total knee arthroplasty of the two groups pre- and post-operatively, and the preand postoperative results within each group.

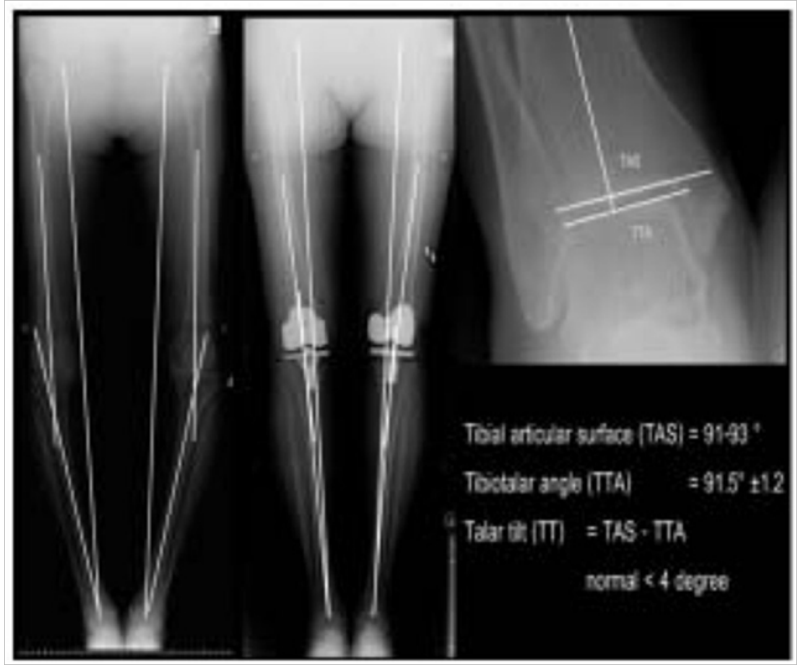

Figure I Pre/Post-operative Mechanical axis and talar tilt angle.

We used the foot functional index (FFI). ${ }^{4}$ scores in clinical assessment pre- and post-operatively, and FFI was compared with talar tilt (TT) for radiologic assessment.

\section{Surgical technique}

Total knee arthroplasty was performed by a single surgeon with a combination of the gap balancing and measured resection techniques using both conventional total knee arthroplasty and computer- assisted total knee arthroplasty. ${ }^{1}$ Tibial cut was performed first, after which the extension and flexion gaps were measured. The distal femoral cut thickness and angle were calculated, and after soft tissue balancing was carried out, femoral block cut was performed. Prosthesis implantation was the final step. Conventional total knee arthroplasty used NexGen LPS prosthesis, Zimmer, and computer-assisted total knee arthroplasty used Emotion knee prosthesis, Asculab, Orthopilot Navigation version 4.3.

\section{Statistics}

We used Descriptive Statistics. Mean, SD for radiographic preoperations and post-operations. For foot functional index we used student t-test and Wilcoxon Signed Ranks Test, and pd" 0.05 was considered statistically significant.

\section{Results}

We enrolled 28 patients (56 total knee arthroplasties) of whom 26 were female and 2 were male and whose mean age was 67.9 year old (the youngest was 58, and the oldest was 82 years old). There were no significant differences in pre-operative TAS and TTA in the two groups (Table 1).

The mean pre-CAS-TKA talar tilt was $0.7 \mathrm{o}$, while in the preconventional TKA it was 1.10 , but these differences were not statistically significant $(\mathrm{p}=0.45)$ as shown in Table 2 ; however, preoperative talar tilt $(1.0 \mathrm{o})$ and post-operative talar tilt $(0.0 \mathrm{o})$ in all cases were significantly different as shown in Table $3(\mathrm{p}=0.02)$.

Table I Number, mean and quality of patient in this serios $(n-56)$.

\begin{tabular}{llllll}
\hline & Age & Pre-op TAS & Pre-op TTA & Pre-op_TAS & Pre-op_TTA \\
\hline Mean \pm SD & $67.96 \pm 679$ & $91.29 \pm 3.63$ & $90.38 \pm 3,99$ & $91.04 \pm 4,45$ & $90.79 \pm 4.39$ \\
Median & 66.50 & 91 & 90 & 90 & 90 \\
Minimum & 58 & 84 & 80 & 84 & 84 \\
Maximum & 82 & 101 & 101 & 112 & 112 \\
\hline
\end{tabular}

TAS:Tibial articular Surface Angle TTA:Talar Angle

Table 2 Mean radiographic measurement: CAS Tic.R vs CCINVTKR.

\begin{tabular}{|c|c|c|c|c|c|}
\hline \multirow{2}{*}{ Computer-Assisted Surgery } & \multicolumn{4}{|c|}{ Conventional method } & \multirow{2}{*}{ p-value } \\
\hline & Mean & S.D & Mean & S.D & \\
\hline \multicolumn{6}{|l|}{ Angle radiographs } \\
\hline Pre_TAS & 90.89 & 3.55 & 91.68 & 3.72 & 0.094 \\
\hline Prc_TTA & 90.18 & 3.88 & 90.57 & 4.17 & 0.562 \\
\hline Post_TAS & 90.71 & 3.68 & 91.36 & 5.15 & 0.369 \\
\hline \multirow[t]{2}{*}{ Post_TTA } & 90.18 & 3.15 & 91.39 & 5.35 & 0.095 \\
\hline & 0.71 & 2.19 & I.II & 3.15 & $0.450 \mathrm{~W}$ \\
\hline Median(Min, Max) & $0.50(-5,8)$ & & I.50(-5,8) & & \\
\hline Post-TT & 0.54 & 1.93 & -0.04 & 1.58 & $0.400 \mathrm{~W}$ \\
\hline Median(Min, Max) & $\mathrm{I} .00(-3,8)$ & & $0.00(-5,3)$ & & \\
\hline
\end{tabular}

p-value from pair t-test; W: p-value from Wilcoxon Signed Ranks Test;TAS:

Tibial Articular Surface angle TTA:Tatar Angle; TT:Talar Tilt = (TAS-TTA)

Table 3 Talar tilt pre vs. post-operation.

\begin{tabular}{llll}
\hline & PPre-op & PPost-op & P-value \\
\hline TT angle Median (Min,Max) & $1.00(-5,8)$ & $0.00(-5,8)$ & 0.026
\end{tabular}


A comparison of pre-operative and post- operative talar tilt (TT) showed that in the CAS-TKA group, talar tilt was not significantly different; however, in the Conventional TKA group there was a significant difference $(\mathrm{p}=0.016)$ as shown in Table 4 .

A comparison of a foot functional index (FFI) for clinical foot and ankle assessment revealed that there was no significant difference between pre- operative foot functional indexes (FFI) in the two groups. However, postoperative pain, difficulty walking (?), and overall subscores were significantly different $(\mathrm{p}$-value $=0.033,0.039$ and 0.047 , respectively) while only scores for daily life activity limitation were not significantly different (Table 5).

Table 4 Talar tilt : pre-op vs, post-op in CASTKR vs CONVTKR

\begin{tabular}{llll}
\hline & Pre op & Post-op & p-value \\
\hline CAS TKR & $0.50(-5,8)$ & $1.00(-3,8)$ & 0.657 \\
Median (Min, Max) & $0.71 \pm 2.19$ & $0.54 \pm 1.93$ & \\
Mean \pm SD. & & & \\
CONVTKR & $1.50(-5.8)$ & $0.00(-5.3)$ & 0.016 \\
Median (MM, Max) & $1.11 \pm 3.16$ & $-0.04 \pm 1.58$ & \\
Mean \pm SD. &
\end{tabular}

Table 5 Foot Functional Index (FFI) CASTKR vs. CONVTKR.

\begin{tabular}{|c|c|c|c|c|c|c|c|}
\hline \multirow[t]{2}{*}{ Clinical assessment } & \multicolumn{3}{|c|}{ Computer assisted surgery } & \multicolumn{3}{|c|}{ Conventional Method } & \multirow{2}{*}{$p$-value } \\
\hline & Median & Min & $\operatorname{Max}$ & Median & Min & Max & \\
\hline \multicolumn{8}{|l|}{ Pre-op } \\
\hline pain & 1.10 & 0.2 & 6 & I.I & 0 & 6.4 & 0.533 \\
\hline difficulty & 1.94 & 0.22 & 6.78 & 1.89 & 0.22 & 6.89 & 0.269 \\
\hline daily life activity limitation & 2.67 & 0 & 8.33 & 2.83 & 0 & 9.33 & 0.604 \\
\hline overall & 1.91 & 0.24 & 6.65 & 1.85 & 0.18 & 6.88 & 0.577 \\
\hline \multicolumn{8}{|l|}{ Post-op } \\
\hline pain & 0.13 & 0.2 & 4.8 & 0.8 & 0.2 & 5 & 0_033* \\
\hline Difficulty & 0.78 & 0.22 & 7 & 1.94 & 0.22 & 7.33 & 0.0391 \\
\hline Daily life activity limitation & 2 & 0 & 9.33 & 2,17 & 0 & 9,33 & 0.32 \\
\hline overall & 1.65 & 0.24 & 6.76 & 1.68 & 0.24 & 7 & $0.047^{*}$ \\
\hline
\end{tabular}

p-value from Wilcoxon Signed Ranks Test, *significant at the 0.05 level

Table 6 Foot Functional Indox (FFI): pro vs. post-CONVTKR

\begin{tabular}{|c|c|c|c|c|c|c|c|}
\hline \multirow[t]{3}{*}{ Clinical assessment } & \multicolumn{6}{|c|}{ Conventional Method } & \multirow[t]{2}{*}{$p$-value } \\
\hline & Pre-op & & & Post-op & & & \\
\hline & Median & Min & Max & Median & Min & Max & \\
\hline pain & I.I & 0 & 6.4 & 0.8 & 0.2 & 5 & I \\
\hline difficulty & 1.89 & 0.22 & 6.89 & 1.94 & 0.22 & 7.33 & 0.314 \\
\hline daily life activity limitation & 2.83 & 0.00 & 9.33 & 2.17 & 0.00 & 9.33 & 0.075 \\
\hline overall & 1.85 & 0.18 & 6.88 & 1.68 & 0.24 & 7.00 & 0.208 \\
\hline
\end{tabular}

Table 7 Foot Functional Index (FFI); pre vs. post-CAS TKR

\begin{tabular}{|c|c|c|c|c|c|c|c|}
\hline \multirow{3}{*}{ Clinical assessment } & \multicolumn{7}{|c|}{ Computer-Assisted Surgery } \\
\hline & \multicolumn{3}{|c|}{ Pre-op } & \multicolumn{3}{|l|}{ Post-op } & \multirow[t]{2}{*}{ p-value } \\
\hline & Median & Min & Max & Median & Min & Max & \\
\hline Pain & I.I & 0.2 & 6 & 0.8 & 0.2 & 4.8 & 0.352 \\
\hline Life difficulty for living & 1.94 & 0.22 & 6.78 & 1.78 & 0.22 & 7 & $0.046^{*}$ \\
\hline Daily life activity limitation & 2.67 & 0.00 & 8.33 & 2.00 & 0.00 & 9.33 & $0.025^{*}$ \\
\hline Overall & 1.91 & 0.24 & 6.65 & 1.65 & 0.24 & 6.76 & $0.047 *$ \\
\hline
\end{tabular}

p-value from Wilcoxon Signed Ranks Test; *significant at the 0.05 level

Clinical assessment of patients in the Conventional TKA group, revealed no significant difference pre- and post-operatively, as shown in Table 6. In contrast, in the CAS-TKA group, pre- and postoperative clinical assessment revealed significant differences in difficulty, daily life activity limitation and overall scores ( $\mathrm{p}$-value $=0.046,0.025$ and 0.047 , respectively), while the only scores that were not significantly different were those for pain (Table 7).

\section{Conclusion}

Ankle osteoarthritis is mostly secondary osteoarthritis that develops as a sequel to fractures and few reports are found of primary osteoarthritis in the ankle. The factors that influence the onset and progression of osteoarthritis are not well understood, although it is known that lower extremity mal-alignment is related to joint problems. ${ }^{2,5-9}$ We found that many patients complained of pain in the ankle after total knee arthroplasty, and we surmised that a change in the alignment of the lower extremity caused by a relatively large knee varus correction angle might be one of the reasons for the discomfort. Jung Hee Lee et al. ${ }^{3}$ reported that $35.2 \%$ of patients had radiographic change in ankle joint after conventional total knee arthroplasty (TKA), and this may be an early sign of ankle osteoarthritic change.

Takakura et al. ${ }^{10}$ reported that varus deformity of the ankle was compensated by the valgus inclination of the subtalar joint, and osteoarthritis began to progress when the compensatory function 
was inoperative. To date, however, the relationship between subtalar compensation and medial compartment osteoarthritis of the ankle has not yet been explored.

According to several cadaveric studies on degenerative changes of the lower extremity, about a third of patients who had osteoarthritis in the knee also had it in the ankle. ${ }^{11-14}$ In particular, Tallroth et al. ${ }^{14}$ analyzed pre- and postoperative radiographs and clearly observed ankle joint osteoarthritis before surgery in 30 out of 104 patients who underwent conventional total knee arthroplasty (TKA).

In our study, we aimed to minimize the alteration of mechanical axis after total knee arthroplasty by utilizing the computer-assisted technique, which we believed, could achieve superior alignment of components, resulting in more accuracy and closer-to- normal knee alignment; in turn, this might minimize the chance of future osteoarthritic change in the ankle.

We measured talar tilt for radiologic evaluation and foot functional index (FFI) for clinical assessment. We found smaller alteration for talar tilt in the computer- assisted group, which we assume, is because this technique helps to minimize ankle problems and is less likely than conventional total knee arthroplasty to lead to ankle osteoarthritic change.

We also found that Foot functional index (FFI) tended to be better in the computer-assisted TKA group than in the conventional TKA one. There were also greater post-operative clinical improvements in the computer-assisted group compared to their pre- operative assessment.

One limitation of this study is that we studied only 28 patients, which is a small number; furthermore, the follow-up period was only of 12 months duration and, therefore, our results may not be very reliable. Another limitation was a lack of equipment: when degenerative changes and angular deformities of the knee are severe, a varus deformity is usually three- dimensionally associated with a flexion contracture of the knee, so the varus angle measured on radiographs may not be a true varus angle. ${ }^{15,16}$ and we did not have CT scan available to evaluate these deformities. With regard to ankle radiographic change, we did not have lateral plain film or CT scan to evaluate other dimensional deformities.

In conclusion, we studied 28 patients in 56 total knee arthroplasty operations, and our study showed that computer-assisted total knee arthroplasty was superior to conventional total knee arthroplasty in minimizing the chance of early postoperative ankle arthritis, but further research is required with larger numbers of patients, longer follow-up and more accurate radiographic imaging applications.

\section{What is already known on this topic?}

1. Some research told after convention TKA had the problem of ankle pain. The hypothesis of acute change of talar tilt angle after TKA.

2. What does this study add?

3. The research shows results of CON TKA compared with CAS TKA in Mechanical alignment of knee in Bilateral TKA.

4. The research shows resulting effect of talar tilt angle post CAS TKA compared with CON TKA.
5. The research shows clinical results of Angle pain after pertoperation CON TKA vs CAS TKA.

\section{Acknowledgments}

This present study was supported by the research fund of Rajavithi Hospital.

\section{Conflicts of interest}

None.

\section{References}

1. Chandler JT, Moskal JT Evaluation of knee and hindfoot alignment before and after total knee arthroplasty: a prospective analysis. $J$ Arthroplasty. 2004;19(2):211-216.

2. Tetsworth K, Paley D Malalignment and degenerative arthropathy. Orthop Clin North Am. 1994;25(3):367-377.

3. Lee JH, Jeong BO Radiologic changes of ankle joint after total knee arthroplasty. Foot Ankle Int. 2012;33(12):1087-1092.

4. Lee WC, Moon JS, Lee HS et al. Alignment of ankle and hindfoot in early stage ankle osteoarthritis. Foot Ankle Int. 2011;32(7):693-699.

5. Budiman-Mak E, Conrad KJ, Roach KE The Foot Function Index: a measure of foot pain and disability. $J$ Clin Epidemiol. 1991;44(6):561-570.

6. Cooke TD, Harrison L, Khan B et al. Analysis of limb alignment in the pathogenesis of osteoarthritis: a comparison of Saudi Arabian and Canadian cases. Rheumatol Int. 2002;22:160-164.

7. Desm D, Galand-Desm S, Besse JL et al. Axial lower limb alignment and knee geometry in patients with osteoarthritis of the knee. Rev Chir Orthop Reparatrice Appar Mot. 2006;92(7):673-679.

8. Guichet JM, Javed A, Russell J, Saleh M Effect of the foot on the mechanical alignment of the lower limbs. Clin Orthop Relat Res. 2003;415:193-201.

9. Mizu-uchi H, Matsuda S, Miura $\mathrm{H}$ et al. The effect of ankle rotation on cutting of the tibia in total knee arthroplasty. $J$ Bone Joint Surg Am. 2006;88(1):2632-2636.

10. Takakura Y, Tanaka Y, Kumai T et al. Low tibial osteotomy for osteoarthritis of the ankle. Results of a new operation in $\mathbf{1 8}$ patients. $J$ Bone Joint Surg Br. 1995;77(1):50-54.

11. Buckwalter JA, Saltzman CL Ankle osteoarthritis: distinctive characteristics. Instr Course Lect. 1999;48:233-241.

12. Huch K, Kuettner KE, Dieppe P Osteoarthritis in ankle and knee joints. Semin Arthritis Rheum. 1997;26(4):667-674.

13. Muehleman C, Bareither D, Huch K et al. Prevalence of degenerative morphological changes in the joints of the lower extremity. Osteoarthritis Cartilage. 1997;5(1):23-37.

14. Tallroth K, Harilainen A, Kerttula L Ankle osteoarthritis is associated with knee osteoarthritis. Conclusions based on mechanical axis radiographs. Arch Orthop Trauma Surg. 2008;128:555-560.

15. Hunt MA, Fowler PJ, Birmingham TB et al. Foot rotational effects on radiographic measures of lower limb alignment. Can J Surg. 2006;49(6):401-406.

16. Cooke D, Scudamore A, Li J et al. Axial lower-limb alignment: comparison of knee geometry in normal volunteers and osteoarthritis patients. Osteoarthritis Cartilage. 1997;5(1):39-47. 